\title{
MODELLING, ANALYSIS AND IMPROVEMENT OF MOBILE BUSINESS PROCESSES WITH THE MPL METHOD
}

\author{
Volker Gruhn, André Köhler \\ University of Leipzig, Applied Telematics/e-Business Group, Klostergasse 3, 04109 Leipzig, Germany \\ \{gruhn, koehler\}@ebus.informatik.uni-leipzig.de
}

Keywords: $\quad$ process modelling, process analysis, mobility

\begin{abstract}
This paper introduces the Mobile Process Landscaping (MPL) method for modelling, analysing and improving mobile business processes. Current approaches for process modelling and analysis do not explicitly allow the consideration of typical mobility issues, e.g. location-dependent activities, mobile networks as resources and specifics of mobile information systems. Thus, our method focuses on the modelling and analysis of these characteristics, and is furthermore based on the process landscaping approach, supporting the easy creation of hierarchical models of distributed processes. The method comes with a specialized modelling notation and guidelines for the creation of process landscapes, context models, and business object models. Furthermore, it provides a catalogue of formally defined evaluation objectives, targeting at typical mobility issues. Each evaluation objective can automatically be tested on the created process landscape. Furthermore, the method includes a best practices catalogue with patterns for process and application improvements for typical mobility situations. A validation of the method is presented showing results from the method's use in a real-world project.
\end{abstract}

\section{Introduction}

The subject of mobile business processes is to perform on-site services, either for external partners of a company or due to internal requirements. Current approaches for modelling, analysing and improving mobile business processes and the supporting mobile applications do not satisfy current industry requirements. This paper introduces the Mobile Process Landscaping (MPL) method in order to close this methodological gap. It leads its user from mobilityspecific evaluation objectives in the beginning to tangible process and application improvements in the end. The method includes a detailed procedure description, a modelling notation, modelling guidelines, an evaluation objectives catalouge, the definition of analysis algorithms, support for result interpretation, and a best practices catalouge for mobile process and applications improvement.

The paper is organized as follows. The method along with a case study from the insurance industry is presented in section 2 . In section 3 , an overview about the related work is given. Section 4 contains a summary and draws a conlusion.

\section{The MPL Method}

Within this chapter, the MPL method is described in more detail. For this purpose it is shown how the method was applied in a real-world case, taken from the insurance industry. Subject of this case study is the assessment of vehicle damages after car accidents by an assessor of an insurance company.

In our case study, the company was not satisfied with the overall performance of the process. Within this situation, the MPL method was applied. In the following sections the four steps of the method are explained by example of this case:

1. Creation of a mobile business process model.

2. Selection of evaluation objectives.

3. Model analysis regarding the choosen evaluation objectives. 


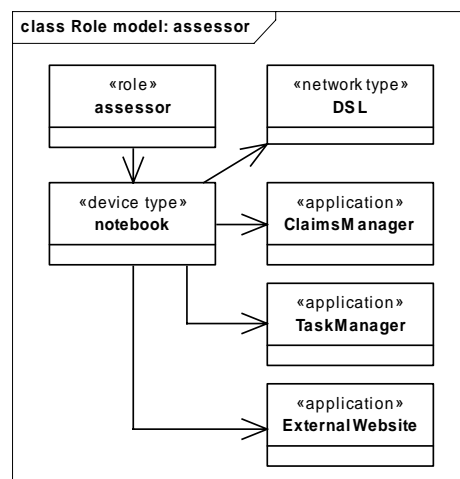

Figure 1: Role model: assessor

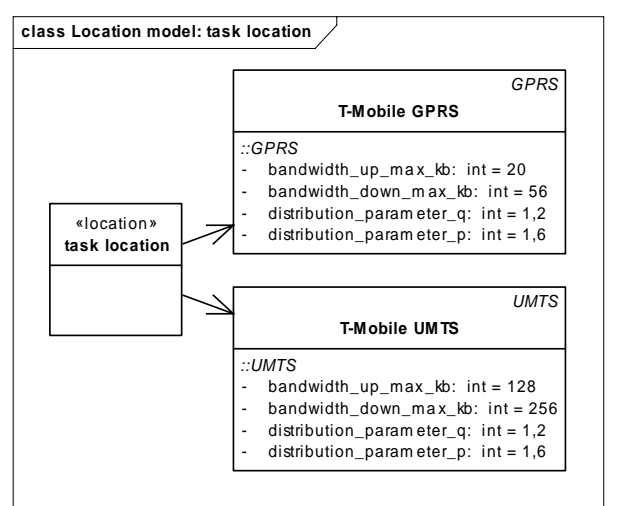

Figure 2: Location model: task location
4. Interpretation of analysis results and deduction of appropriate activities.

\subsection{Method Step 1: Creation of a Mobile Business Process Model}

The model created during the use of the MPL method is called the mobile business process model. It consists of a process context model, a business object model and a process landscape model.

\subsubsection{Process Context Model}

A mobile business process is executed within a context, which is of particular relevance for the process evaluation. Such context information cannot be modelled as a process structure. Thus, a separate model is created which contains:

- roles that a person can act as,

- locations at which a process step is executed,

- applications that a role can use within in a process step,

- devices necessary for running the applications,

- services provided by an application, and

- networks available at some location and required by applications.

The MPL method provides a metamodel for the creation of the process context model, which is constituted as UML class diagrams (Figure 1 and 2 show examples).

\subsubsection{Business Object Model}

The construction of business object models is a wellknown approach and will not be described here in detail (see e.g. (Eeles and Sims, 1998)). The model should contain all business objects exchanged within the process as well as their relationships to each other. It is realized as one or more UML class diagrams.

\subsubsection{Process Landscape Model}

During earlier work, we introduced the process landscaping approach (Gruhn and Wellen, 2001), (Gruhn and Wellen, 2000). It pays particular attention to the modelling of distributed processes with emphasis on the data and documents moving around between different locations. The strength of this approach is that it consists of hierarchically structured process models and interfaces between them, which can be described at different levels of detail. It turned out that this approach is very useful for modelling mobile business processes. Thus, it is used in the presented method.

A mobile process landscape is modeled as an overview of the mobile business processes of an organization. A process landscape is realized as one process model, containing process steps that can be refined by other process models or application interaction models on demand. A process model describes what the mobile worker is doing (business view). An application interaction model describes, how the mobile worker is using mobile technologies within a process step (application view). As modelling notation for process landscapes coloured petri nets (CPN) are used (Jensen et al., 2007). They allow the graphical modelling of control and object flows, have a strong formal basis and are thus ideal for static and dynamic analysis purposes. Furthermore, a couple of standard analysis techniques are available.

A cut-out of the process landscape model for the insurance industry case is given in Figure 3, showing the top-level of the process landscape. Each activity in this model is refined by another process model, describing the refined activity in more detail. Within a process model the attributes role and location must be 


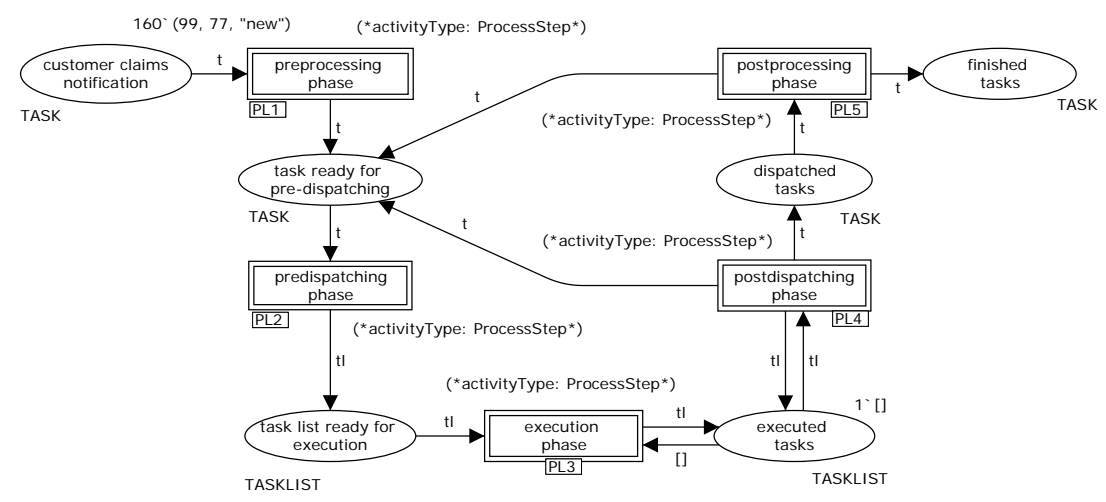

Figure 3: Root model for case study

specified for each process step, indicating which role is executing the process step at which possible locations. For each application interaction, the attribute required connection class must be specified, indicating which type of network connection is required. The attribute values are necessary for the later model evaluation and must match the before described context model.

Regarding the claims assessor case, it turned out that the creation of consistent context, business object and process landscape models required significiant modelling effort. The reason was the unexpectedly different perception of the process context, business terms, and process structures in the project group. The resulting discussion led to a significantly better, more detailed and commonly agreed understanding of the process and its contexts. This knowledge gain, resulting from the MPL method use, was a nice additional benefit.

\subsection{Method Step 2: Selection of Evaluation Objectives}

The second step in the MPL method is the selection of evaluation objectives. Current process modelling methods and tools offer a large number of general evaluation objectives, e.g. identification of deadlocks, measuring time and costs and many more (Giaglis, 2001), (Hommes and Van Reijswoud, 2000). However, to achieve as meaningful results as possible, the specific process characteristics need to be considered during process evaluation. Compared to nonmobile business processes, mobile ones are usually more difficult to manage in certain aspects. They are expensive due to mobility costs and complicated to plan because of place, time and resource restrictions. Furthermore, mobile processes often do not possess the necessary flexibility for an appropriate reaction to suddenly changed situations on-site. Thus, the MPL method provides a catalogue of evaluation objectives, purpose-built for the analysis of mobile business processes (see Table 1).

For each evaluation objective (EO), the catalogue contains an in-depth description of the evaluation goal, a description of how to perform the model analysis, and a description of the expected results along some interpretation guidelines. A detailed presentation of the catalogue itself is beyond the scope of this paper. Nonetheless, Table 2 gives an impression of how an objective is defined by example of EO.1.1.

\subsection{Method Step 3: Model Analysis Regarding the Choosen Evaluation Objectives}

Once the business process model is created and the desired evaluation objectives are choosen, the model evaluation can start. As a real-world model consists easily of a couple of hundred activities, distributed over hierachical submodels, the evaluation can hardly be executed manually. Thus, appropriate tool support comes with the MPL method, allowing to

- check the CPN model against the MPL modelling guidelines,

- check integrity between context, business object, and process landscape models, and

- check the CPN model against evaluation objectives.

However, the scope of this paper is not to illustrate the tool support, but to present how the process model can be analysed. This will be done by example of the claims assessor case. For the sake of brevity, the analysis results are described only for the evaluation objectives EO.1.1. As described in the definition 
EO.1 Process Distribution Objectives

EO.1.1 Distribution by execution location

EO.1.2 Distribution by executing role

EO.2 Process Performance Objectives

EO.2.1 Mobile idle periods of information objects

EO.2.2 Required time to process completion

EO.3 IT-support Objectives

EO.3.1 Availability of applications and data

EO.3.2 Timeliness of applications and data

EO.3.3 Process robustness in offline situations

Table 1: Evaluation objectives catalogue

\begin{abstract}
Objective identifier. EO.1.1 Distribution by execution location Objective motivation. Different process parts are executed at different locations. Each location change causes efforts and requires time. The more location changes occur within a process, the higher is its potential for optimzation.

Objective goal. To evaluate the fragmentation of the process model regarding location changes.

Model preparation. A location transition graph is required.

Model evaluation. To identify sequences in the location transition graph, containing the same mobile location node, separated by one or more stationary nodes, at least two times.

Evaluation result. List with sequences fulfilling the above given definition.

Usage of evaluation result. It should be tried to avoid the identified sequences by manual process optimization.
\end{abstract}

Table 2: Excerpt from evaluation objectives catalogue (by example of EO.1.1) of these analysis (see Table 2), the creation of a location transition graph is required. A location transition graph is a reduction of the original process model. A set of connected activities from the process model executed at the same location is reduced to a single node in the location transition graph. Each arc between these nodes stands for a location change.

Figure 4 shows the location transition graph for the claims assessor case. A node in the location transition graph can represent a stationary location $(S L)$ or a mobile location $(M L)$. Nodes with the same label in the location transition graph represent different parts of the process model, their included activities are to be executed at the same location.

The first node $S L_{1}$ stands for a group of connected activities to be executed in the backoffice of the company (preprocessing phase). The following node $S L_{2}$ stands for a group of activities to be executed in the dispatcher's office (predispatching phase), node $S L_{3}$ contains the activities to be executed in the home office of the mobile worker (part of execution phase). These nodes represent activities from different stationary locations, whereas node $M L_{1}$ represents activities from the task location, which is a mobile location (execution phase). This model part is followed by a another group of activities to be executed in the home office of the mobile worker. Subsequently, a group of activities to be executed at the dispatchers office (postdispatching phase) and the backoffice (postprocessing phase) follow. Arcs between these nodes represent possible locations transitions in the process model.

The subject of the analysis EO.1.1 is to evaluate how strong the process model is fragmented regarding location changes (see Table 2). The evaluation goal is to identify sequences in the location transition graph, which contain the same mobile location node, sepa- rated by one or more stationary nodes, at least two times. The results of analysis EO.1.1 are illustrated in Figure 4. Two sequences were found. The first sequence shows the case that after task execution the task is getting reassigned by the dispatcher. This can be necessary, if the task state or the produced result does not match the dispatchers requirements. This results in the fact that the mobile task passes the model area $M L_{1}$ a second time. The second sequence has a similar reason. The customer agent can also reassign the task to the mobile worker, due to the same reason and with same consequences.

This analysis identified two weak points in the process model, which are typical mobility problems. These recurrent visits are necessary, if the mobile worker can not accomplish his task due to missing ressources or suddenly changed requirements at the point of service. Recurrent visits cause usually high costs and a long process duration and should thus be avoided if possible.

\subsection{Method Step 4: Interpretation of Analysis Results and Deduction of Appropriate Activities}

The MPL method does not leave the user alone with the evaluation results, but provides a catalogue of best practices for changing process structures and the supporting applications in order to improve the mobile business processes regarding the incurring process costs, the required time for process completion and the quality of the process outcome. The best practices are extracted from related work and are described in the well-known pattern approach (see, e.g., (Rising, 1998))

To present this catalogue is beyond the scope of 


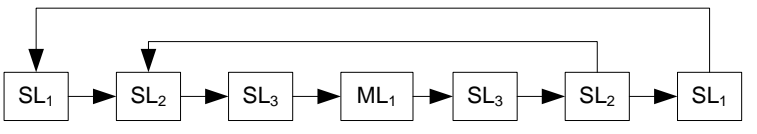

$\mathrm{SL}_{1}$ backoffice

$\mathrm{SL}_{2}$ dispatcher office

$\mathrm{SL}_{3}$ home office

$\mathrm{ML}_{1}$ task location

\begin{tabular}{ll}
\hline$\#$ & Sequence \\
\hline 1 & $M L_{1} \rightarrow S L_{3} \rightarrow S L_{2} \rightarrow S L_{2} \rightarrow S L_{3} \rightarrow M L_{1}$ \\
2 & $M L_{1} \rightarrow S L_{3} \rightarrow S L_{2} \rightarrow S L_{1} \rightarrow S L_{1} \rightarrow S L_{2} \rightarrow S L_{3} \rightarrow$ \\
& $M L_{1}$ \\
\hline
\end{tabular}

Figure 4: Location transition graph and evaluation results for EO.1.1

this paper. Nonetheless, the induced activities regarding the above described evaluation results will be illustrated. Regarding the situation of the recurrent visits (EO.1.1), an analysis of the activating transitions lead to the result, that the mobile worker may not be able to find the damaged car's location due to missing informations within the task description, especially if the car's location is different from the address of the insurance holder. Thus, the application used for the generation of the tasks was modified to include the customer's home address as well as the car's location address. The recording of the latter was inserted as an activity into the initial service agent's process. Through this addition, the incidence rate of recurrent visits could be reduced by nearly half.

This illustration is just a small section of the whole analysis result and the induced improvement actions. The results caused an extensive and detailed discussion about possible process and application improvements in the company. In conclusion, a bundle of actions was created to apply a large number of the discussed changes. Finally, after completion of these actions, the company could observe significant improvements in process costs and time. Thus, it could be shown that the use of the MPL method was benefical for the improvement of mobile business processes in this case.

\section{Related Work}

Barnes gives a taxonomy as well as examples for mobile distributed work (Barnes, 2004). The taxonomy framework allows to classify mobile business cases. It consists of the dimensions mobility (transient, mobile, remote), process (automation, decision support, transformation) and value proposition (mobile channel access, mobile service value, mobile service creation). This framework is a good starting point if a company begins with a first evaluation of their mobile business processes. Innes et al. present an application of this framework for trade services organisations (Innes et al., 2005).

Yuan and Zheng also provide a framework for analysing mobile work support (Yuan and Zheng, 2005). The framework consists of the dimensions worker, task, context, and technology. With this framework characteristics of stationary and mobile work are compared in order to better understand mobile work situations. An extension of this framework is presented in (Yuan and Zheng, 2006), focusing on the fit between mobile task and mobile technology types. Perry et al. (Perry et al., 2001) also present results regarding the basic concept of mobility. They present a study where different aspects of mobile work in general are examined. The four key factors identified for mobile work are the role of planning, working in dead time, accessing remote resources and monitoring distant activities.

Giaglis proposes an evaluation framework for business process modelling and information systems modelling techniques (Giaglis, 2001). It consists of three evaluation variables: the breadth (typical modelling goals and objectives), the depth (functional, behavioral, organizational, and informational perspective), and the fit (typical project types the technique could support). When applying this framework to the MPL method, the result is that the method has a limited operational area (i.e. process development and improvement). Furthermore, it turned out that the MPL method supports the whole depth dimension (support of functional, behavioral, organizational, and informational perspective). Combining these results, the fit dimension gives projects types where the MPL method could be used. Despite others they are: system analysis and design, business process reengineering, task redesign, and workflow design.

A comprehensive study of methodologies, techniques, and tools for business process change is presented by Kettinger et al. (Kettinger and Teng, 1997). The authors examined nearly all available methods and tools in an extensive research project. The main result is a classification scheme, suitable for discovering the right method or tool for a certain purpose. Within this scheme, the MPL method belongs to the stages 3: Diagnose (document existing process, analyze existing process), and 4: Redesign (define and analyze new process concepts, prototype and detailed 
design of new process, analyze and design information systems).

\section{Summary and Conclusion}

This paper presented the MPL method, which aims to model, analyse and improve mobile business processes. We have shown that current approaches do not consider mobility adequately. But, since many industries have to deal with such processes, a specialised method is required. Therefore, we presented the MPL approach that provides a catalogue of predefined evaluation objectives and support for the creation of appropriate models. The evaluation of the resulting model can be automated, leading to substantial results that show possible process and application improvements. The method was applied to a process improvement project from the insurance industry. It was shown that the intended benefits could be realized. Nonetheless, the method has also a couple of limitations. Firstly, the modelling requires a person having strong skills in UML and CPN. Secondly, the quality of the produced results depends heavily on the model quality. An experienced modeler with the evaluation objectives catalogue in mind will most likely produce good models, while an unexperienced firsttime modeler may not. Thirdly, the method produces - as all process modelling approaches - a relatively inflexible model due to the nature of sequences, decsion nodes, and so on. But especially mobile business processes usually allow a great flexibility at the point of service, i.e., being able to do anything anytime and anywhere. Further research is planned to extend this method. Currently, the tool support is still in the prototype stage. The development of a more convenient tool would be useful. Also, the evaluation objectives catalogue has potential for extension.

\section{Acknowledgments}

The Applied Telematics/e-Business Group is endowed by Deutsche Telekom AG. We would like to thank Matthias Book and Ralf Laue for their valuable comments on this paper.

\section{REFERENCES}

Barnes, S. J. (2004). Wireless support for mobile distributed work: A taxonomy and examples. In Proceedings of the Proceedings of the 37th Annual Hawaii In- ternational Conference on System Sciences, pages 30078.1+. IEEE Computer Society.

Eeles, P. and Sims, O. (1998). Building business objects. John Wiley \& Sons, Inc., New York, NY, USA.

Giaglis, G. M. (2001). A taxonomy of business process modeling and information systems modeling techniques. International Journal of Flexible Manufacturing Systems, 13(2):209-228.

Gruhn, V. and Wellen, U. (2000). Structuring complex software processes by process landscaping. In Software Process Technology, Lecture Notes In Computer Science, Vol. 1780, pages 138-149, Heidelberg, Germany. Springer.

Gruhn, V. and Wellen, U. (2001). Process landscaping: Modelling distributed processes and proving properties of distributed process models. In Unifying Petri Nets, Lecture Notes In Computer Science, Vol. 2128, pages 103-125. Springer, London, UK.

Hommes, B. J. and Van Reijswoud, V. (2000). Assessing the quality of business process modeling techniques. In Proceedings of the 33rd Hawaii International Conference on System Sciences, pages 1007+. IEEE Computer Society.

Innes, D., Barnes, S. J., and Scornavacca, E. (2005). The impact of wireless field force automation on new zealand trade services organizations. In Proceedings of the International Conference on Mobile Business, pages 49-55. IEEE Computer Society.

Jensen, K., Kristensen, L., and Wells, L. (2007). Coloured petri nets and cpn tools for modelling and validation of concurrent systems. International Journal on Software Tools for Technology Transfer (STTT), 9(3):213254

Kettinger, W. J. and Teng, J. T. C. (1997). Business process change: a study of methodologies, techniques, and tools. MIS Quarterly, 21(1):55-80.

Perry, M., O'Hara, K., Sellen, A., Brown, B., and Harper, R. (2001). Dealing with mobility: understanding access anytime, anywhere. ACM Transactions on ComputerHuman Interaction, 8(4):323-347.

Rising, L., editor (1998). The Patterns Handbook: Techniques, Strategies, and Applications. SIGS Reference Library. Cambridge University Press.

Yuan, Y. and Zheng, W. (2005). From stationary work support to mobile work support: A theoretical framework. In Proceedings of the International Conference on Mobile Business, pages 315-321. IEEE Computer Society.

Yuan, Y. and Zheng, W. (2006). The fit between mobile task and mobile work support: A theoretical framework. In Proceedings of the International Conference on Mobile Business, pages 11+. IEEE Computer Society. 